\title{
EFICÁCIA DE HERBICIDAS NA DESSECAÇÃO E NO CONTROLE RESIDUAL DE PLANTAS DANINHAS NO SISTEMA DESSEQUE E PLANTE
}

\author{
Erivelton Scherer Roman ${ }^{1}$
}

'Pesquisador. Embrapa Trigo. Caixa Postal 451. Passo Fundo, RS 99001-970 eroman@cnpt.embrapa.br

\begin{abstract}
RESUMO
Os efeitos de chlorimuron-ethyl, na dose de $10,0 \mathrm{~g} / \mathrm{ha}$, aplicado isoladamente e em mistura ou em seqüência a glyphosate, e de diclosulam na dose de 35,2 g/ha em mistura de tanque com glyphosate, e capina, foram estudados para o controle de Brachiaria plantaginea (Link) Hitchc. e Bidens pilosa L. em soja, no sistema desseque e plante, em plantio direto. Foi avaliada, também, a segurança dos tratamentos à cultura. O trabalho foi conduzido, em campo, durante o ano agrícola 2001/ 2002, na área experimental da Embrapa Trigo, em Passo Fundo, RS. O delineamento experimental usado foi de blocos completamente casualizados, com 4 repetições. Todos os tratamentos químicos utilizados foram eficientes na dessecação de B. pilosa. Chlorimuron-ethyl e diclosulam, nas doses testadas, aplicados sozinhos e em mistura de tanque ou em seqüência à glyphosate, propiciaram efeito residual suficiente para controlar B. pilosa durante o ciclo da soja. Não foram observados sintomas tóxicos à cultura, nem efeito dos tratamentos químicos sobre o rendimento de grãos.
\end{abstract}

Palavras-chave: Bidens pilosa, Brachiaria plantaginea, chlorimuron-ethyl, diclosulam, glyphosate.

\section{ABSTRACT \\ Herbicide efficacy and selectivity for burn-down and residual weed control in soybeans}

The effects of chlorimuron-ethyl at the rate of $10.0 \mathrm{~g}$ /ha applied alone and as tank mixture or in sequence with glyphosate, and diclosulam at the rate of $35.2 \mathrm{~g} / \mathrm{ha}$ plus glyphosate, as tank mixture, were studied for burn-down and residual control of Brachiaria plantaginea (Link) Hitchc. and Bidens pilosa L. and for their safety to soybean in no-till. The study was conducted during the 2001/2002 growing season at the National Wheat Research Center of Embrapa in Passo Fundo, RS, Brazil, on a medium-textured soil. The experimental design was a completely randomized block with four replications. All treatments were effective in desiccating B. pilosa. Chlorimuron-ethyl and diclosulam, applied alone, in mixture or in sequence with glyphosate provided season long weed control. No crop injury was observed and there was no effect on grain yield.

Key words: Bidens pilosa, Brachiaria planstaginea, chlorimuron-ethyl, diclosulam, glyphosate. 


\section{INTRODUÇÃO}

No sistema de manejo convencional de solo, as plantas daninhas presentes por ocasião do preparo do solo são controladas mecanicamente pelos implementos que realizam os cultivos. No plantio direto esse trabalho é, geralmente, realizado por herbicidas dessecantes, os quais devem ser, no mínimo, tão eficientes quanto o preparo do solo no controle das plantas daninhas presentes, para evitar a competição com a cultura (Roman \& Didonet, 1990).

Herbicidas à base de 2,4-D são usados em combinação com o glyphosate para ampliar o espectro de espécies de plantas daninhas controladas por esse produto. No entanto, o 2,4-D, especialmente na formulação éster, pode apresentar problemas de deriva e volatilização, causando danos às culturas sensíveis localizadas nas proximidades. Para segurança da cultura de soja, é recomendada a observância de um intervalo de dez dias entre a aplicação e o plantio (Reunião...,1994). Além disso, esses produtos não apresentam efeito sobre o banco de sementes das plantas daninhas (Ashton \& Crafts, 1981). A utilização de herbicidas no manejo, que permitam efeito residual no solo, no sistema chamado "desseque e plante", pode ser uma alternativa para reduzir infestações de plantas daninhas na cultura, causando redução dos custos de controle dessas espécies (Carvalho et al., 2000). Esse sistema consiste na aplicação de herbicidas seguida pelo plantio de soja, sem necessidade de intervalo de tempo entre essas práticas.

O objetivo do presente estudo foi avaliar o efeito de herbicidas aplicados como dessecantes, para controle de plantas daninhas em plantio direto de soja.

\section{MATERIAL E MÉTODOS}

O experimento foi conduzido em condições de campo na área experimental da Embrapa Trigo, localizada no município de Passo Fundo, RS, na safra 2001/2002. O solo é de textura média, com $42,0 \%$ de argila e $4,0 \%$ de matéria orgânica, pertencente à Unidade de Mapeamento Passo Fundo (Latossolo Vermelho distrófico típico) . Os tratamentos (Tabela 1) foram aplicados em pré-plantio no dia 16/11/2001, no sistema "desseque e plante". Foram incluídos como padrões, entre esses, os tratamentos com glyphosate+diclosulam, em mistura no tanque, pela ação dessecante e residual, e glyphosate+2,4-D, por ser um tratamento usado amplamente na pulverização de manejo (Rodrigues, 1995). O plantio foi realizado no dia 18/11/2001. A aplicação foi realizada usando-se pulverizador costal de precisão, com pressão de trabalho de $1,1 \mathrm{kgf} / \mathrm{cm}^{2}$ dada por gás carbônico, munido de bicos de jato em forma de leque, tipo 110.015 , espaçados $0,5 \mathrm{~m}$ entre si e posicionados a $50 \mathrm{~cm}$ acima das plantas. $\mathrm{O}$ volume de calda usado foi de $100 \mathrm{~L} / \mathrm{ha}$. O delineamento experimental foi de blocos ao acaso, com 4 repetições. As parcelas mediam $4 \mathrm{~m} \times 6 \mathrm{~m}$.

A cultivar de soja reagente foi BRS 154 , semeada na densidade de 28 plantas por metro quadrado, em sistema de plantio direto. A adubação de soja foi realizada utilizando-se $250 \mathrm{~kg} /$ ha de adubo, fórmula NPK 05-25-25.

As plantas daninhas presentes no experimento, por ocasião da aplicação dos tratamentos, eram Brachiaria plantaginea (Link) Hitchc. e Bidens pilosa L., nas densidades populacionais de 20 e 300 plantas $/ \mathrm{m}^{2}$, respectivamente.

A eficácia dos tratamentos na dessecação de $B$. plantaginea e B. pilosa foi determinada aos 20 dias após o

Tabela 1. Tratamentos utilizados em pré-emergência, no sistema de aplicação desseque e plante, para o controle de Brachiaria plantaginea e de Bidens pilosa. Passo Fundo, RS, 2001-2002.

\begin{tabular}{|c|c|c|c|c|}
\hline \multicolumn{2}{|c|}{ Tratamento } & \multirow[b]{2}{*}{$\begin{array}{c}\text { Forma } \\
\text { de aplicação }\end{array}$} & \multicolumn{2}{|c|}{ Dose } \\
\hline Nome técnico & Nome comercial & & $\begin{array}{l}\text { Prin cípio ativo } \\
\text { (g/ha) }\end{array}$ & $\begin{array}{c}\text { Produto } \\
\text { comercial } \\
\text { (L ou g/ha) }\end{array}$ \\
\hline Chlorimuron-ethyl & Classic & Isolada & 10 & $40 \mathrm{~g}$ \\
\hline Glyphosate & Polaris & Isolada & 720 & $2,0 \mathrm{~L}$ \\
\hline $\begin{array}{l}\text { Glyphosate }+ \\
\text { chlorimuron-ethyl }\end{array}$ & Polaris + Classic & Mistura em tan que & $720+10$ & $2,0 \mathrm{~L}+40 \mathrm{~g}$ \\
\hline $\begin{array}{l}\text { Glyphosate }+ \\
\text { chlorimuron-ethyl }\end{array}$ & Polaris + Classic & $\begin{array}{l}\text { Aplicação em } \\
\text { seqüência }\end{array}$ & 720 e 10 & $2 \mathrm{~L} \mathrm{e} 40 \mathrm{~g}$ \\
\hline $\begin{array}{r}\text { Glyphosate }+ \\
\text { diclosulam }\end{array}$ & Polaris + Spider & Mistura em tanque & $720+35,2$ & $2 \mathrm{~L}+42 \mathrm{~g}$ \\
\hline $\begin{array}{l}\text { Glyphosate }+ \\
2,4-D \text { éster }\end{array}$ & Polaris + Deferon & Mistura em tanque & $720+400$ & $2,0 \mathrm{~L}+1,0 \mathrm{~L}$ \\
\hline Capina & - & - & - & - \\
\hline Testemunha & - & - & - & - \\
\hline
\end{tabular}

(1) No mesmo dia. 
tratamento (DAT), e no controle residual, aos 35 e 45 DAT e em pré-colheita, pelo método de avaliação visual, atribuindo-se notas, em percentagem de controle em relação à testemunha. A escala empregada variou entre 0 (sem danos visíveis) e $100 \%$ (morte total de plantas). A avaliação de toxicidade às plantas de soja foi realizada visualmente aos 20, 35 e aos 45 DAT, e os resultados expressos em percentagem de danos, tomando como base a redução do crescimento, a clorose e a queima da parte aérea foliar (Lich et al., 1997).

Dados de eficácia dos tratamentos e de rendimento de grãos de soja, obtidos na área útil de $16,2 \mathrm{~m}^{2}$ em cada unidade experimental, foram submetidos à análise de variância e as médias dos tratamentos comparadas pelo teste de Duncan, a $5 \%$ de probabilidade de erro.

\section{RESULTADOS E DISCUSSÃO}

\section{Controle de Brachiaria plantaginea}

A avaliação realizada aos 20 DAT indicou que, com exceção do tratamento com chlorimuron-ethyl, aplicado isoladamente, os demais tratamentos foram eficientes na dessecação da espécie, proporcionando $100 \%$ de controle (Tabela 2). A ação relativamente fraca desse composto sobre as gramíneas, quando aplicado em pós-emergência, pode ser devida à habilidade dessa espécie em metabolizar o produto, com rápida conversão a produtos inativos (Zimdahl, 1993; Thill, 1994).

Como a planta daninha não apresentou novos fluxos de emergência, não foram realizadas avaliações do efeito residual dos tratamentos para o controle da espécie.

\section{Controle de Bidens pilosa}

A avaliação realizada aos 20 DAT indicou que todos os tratamentos foram eficientes na dessecação de B. pilosa, proporcionando $100 \%$ de controle (Tabela 3 ).

O controle residual, avaliado aos 35 DAT, propiciado pelos tratamentos com chlorimuron-ethyl e diclosulam, equivaleram-se estatisticamente entre si e com a capina, com níveis de controles superiores a $98 \%$. Aos 45 DAT, os tratamentos com chlorimuron-ethyl e diclosulam propiciaram controles de 95 e $96 \%$, respectivamente. No entanto, foram estatísticamente inferiores à capina, que propiciou $100 \%$ de controle da espécie. Na avaliação realizada em pré-colheita, esses tratamentos equivaleram-se estatísticamente entre si e com a capina, com níveis superiores a $90 \%$ de controle, considerado como "controle" pela Sub-Comissão de Plantas Daninhas da Comissão de Pesquisa de Soja dos estados do Rio Grande do Sul e de Santa Catarina (Reunião...,1994). Nos tratamentos em que chlorimuron-ethyl e diclosulam foram testados em mistura em tanque com glyphosate ou em aplicações em seqüência a esse herbicida, o controle da planta daninha durante o ciclo da cultura foi atribuído ao efeito residual de chlorimuron-ethyl e de diclosulam. Resultados semelhantes foram relatados por Bizzi \& Bianchi (2000), por Valente \& Ornelas (2000) e por Carvalho et al. (2000). A reinfestação de $B$. pilosa no tratamento com glyphosate, aplicado isolado, foi atribuída à falta de efeito residual do produto (Rodrigues, 1995).

O controle residual de plantas daninhas dado por herbicidas aplicados juntamente com herbicidas dessecantes é importante, pois além de melhorar o controle nessa operação, pode reduzir a competição com a cultura durante o período crítico, facilitando o controle por herbicidas pós-emergentes, como observado por Carvalho et al. (2000).

Tabela 2. Controle, aos 20 (dias após o tratamento), de Brachiaria plantaginea, na pulverização de manejo. Embrapa Trigo, Passo Fundo, RS, 2001-2002.

\begin{tabular}{lcc}
\hline \multicolumn{1}{c}{ Tratamento } & & Controle $^{(1)}$ \\
& Nome técnico & $\begin{array}{c}\text { Dose } \\
(\mathbf{g} / \mathbf{h a})\end{array}$ \\
\hline Chorimuron-ethyl & 10 & $15 \mathrm{~b}$ \\
Glyphosate & 720 & $100 \mathrm{a}$ \\
Glyphosate + chlorimuron-ethyl & $720+10$ & $100 \mathrm{a}$ \\
Glyphosate e chlorimuron-ethyl (em seqüência) & $720 \mathrm{e} 10$ & $100 \mathrm{a}$ \\
Glyphosate + diclosulam & $720+35,2$ & $100 \mathrm{a}$ \\
Glyphosate + 2,4-D éster & $720+400$ & $100 \mathrm{a}$ \\
Capina & - & $100 \mathrm{a}$ \\
Testemunha & - & $0 \mathrm{c}$ \\
\hline C.V. $(\%)$ & & 1,9 \\
\hline
\end{tabular}

(1)Valores seguidos pela mesma letra, na coluna, não diferem entre si pelo teste de Duncan, ao nível de probabilidade de erro de $5 \%$. 
Erivelton Scherer Roman

Tabela 3. Controle de Bidens pilosa na cultura de soja. Embrapa Trigo, Passo Fundo, RS, 2001-2002.

\begin{tabular}{|c|c|c|c|c|c|}
\hline \multicolumn{2}{|c|}{ Tratam ento } & \multicolumn{4}{|c|}{ Controle ${ }^{(1)}(\%)$} \\
\hline \multirow[b]{2}{*}{ Nome técnico } & \multirow{2}{*}{$\begin{array}{c}\text { Dose } \\
(\mathbf{g} / \mathbf{h} \mathbf{a})\end{array}$} & \multirow[b]{2}{*}{ Dessecação } & \multicolumn{3}{|c|}{ Residual } \\
\hline & & & 30 DAT & $45 \mathrm{DAT}$ & $\begin{array}{c}\text { Pré- } \\
\text { colheita }\end{array}$ \\
\hline Chorim uron - ethyl & 10 & 100 & 99 a & $95 \mathrm{~b}$ & 93 a \\
\hline Glyphosate & 720 & 100 & $0 \mathrm{~b}$ & $0 \mathrm{c}$ & $0 \mathrm{~b}$ \\
\hline $\begin{array}{l}\text { Glyphosate + chlorimuron- } \\
\text { ethyl }\end{array}$ & $720+10$ & 100 & 99 a & $95 \mathrm{~b}$ & 91 a \\
\hline $\begin{array}{l}\text { Glyphosate e chlorimuron- } \\
\text { ethyl (em seqüên cia) }\end{array}$ & 720 e 10 & 100 & $100 \mathrm{a}$ & $95 \mathrm{~b}$ & 91 a \\
\hline Glyphosate + diclosulam & $35,2+720$ & 100 & $100 \mathrm{a}$ & $96 \mathrm{~b}$ & 93 a \\
\hline Glyphosate $+2,4-\mathrm{D}$ éster & $400+720$ & 100 & $0 \mathrm{~b}$ & $0 \mathrm{c}$ & $0 \mathrm{~b}$ \\
\hline Capin a & $\cdot$ & 100 & 100 a & $100 \mathrm{a}$ & 95 a \\
\hline Testem unh a & - & 0 & $0 \mathrm{~b}$ & $0 \mathrm{c}$ & $0 \mathrm{~b}$ \\
\hline C.V. $(\%)$ & & - & 1,9 & 1,5 & 2,9 \\
\hline
\end{tabular}

(1)Valores seguidos pela mesma letra, na coluna, não diferem entre si pelo teste de Duncan, ao nível de probabilidade de erro de $5 \%$.

\section{Fitotoxicidade}

Não foram observados sintomas tóxicos causados pelos tratamentos em plantas de soja, indicando que todos os tratamentos foram seguros à cultura.

\section{Rendimento de grãos}

Os dados de rendimento de grãos são apresentados na Tabela 4. O rendimento mais baixo foi obtido no tratamento com chlorimuron-ethyl, aplicado isoladamente, o qual não diferiu estatísticamente do rendimento obtido nos tratamentos com glyphosate, glyphosate $+2,4-\mathrm{D}$ éster e na testemunha. $\mathrm{O}$ baixo rendimento nesses tratamentos foi atribuído à interferência das plantas daninhas, uma vez que, de acordo com Rodrigues (1995), glyphosate não apresenta efeito resi- dual e chlorimuron apresenta baixa eficácia para o controle de $B$. plantaginea.

O maior rendimento de grãos foi obtido nos tratamentos capina, glyphosate + diclosulam, em mistura no tanque, e glyphosate mais chlorimuron-ethyl, em seqüência, os quais não diferiram estatísticamente entre si.

\section{CONCLUSÕES}

Nenhum dos tratamentos testados causou toxicidade à soja, nem causou efeito sobre o rendimento de grãos da cultura. O rendimento mais baixo obtido nos tratamentos com chlorimuron-ethyl, glyphosate, glyphosate $+2,4-\mathrm{D}$ éster e na testemunha, foram atribuídos à interferência das plantas daninhas.

Tabela 4. Rendimento de grãos de soja em resposta à aplicação de herbicidas no sistema desseque e plante, em plantio direto. Embrapa Trigo, Passo Fundo, RS, 2001-2002.

\begin{tabular}{|c|c|c|}
\hline \multicolumn{2}{|l|}{ Tratamento } & \multirow{2}{*}{$\begin{array}{l}\text { Rendimento de grãos } \\
\qquad(\mathrm{kg} / \mathrm{ha})\end{array}$} \\
\hline Nome técnico & $(\mathrm{g} / \mathrm{h} \mathbf{a})$ & \\
\hline Chorimuron - ethyl & 10 & $734 \mathrm{~b}$ \\
\hline Glyphosate & 720 & $1007 \mathrm{~b}$ \\
\hline Glyphosate + chlorimuron-ethyl & $720+10$ & $1996 \mathrm{a}$ \\
\hline Glyphosate e chlorimuron-ethyl (em seqüência) & 720 e 10 & 1942 a \\
\hline Glyphosate + diclosulam & $720+35,2$ & $2260 \mathrm{a}$ \\
\hline Glyphosate $+2,4-D$ éster & $720+400$ & $847 \mathrm{~b}$ \\
\hline Capina & & $2173 \mathrm{a}$ \\
\hline Testemunha & & $680 \mathrm{~b}$ \\
\hline C. V. $(\%)$ & & 21,9 \\
\hline
\end{tabular}

${ }^{(1)}$ Valores seguidos pela mesma letra, na coluna, não diferem entre si pelo teste de Duncan, ao nível de probabilidade de erro de $5 \%$. 
Com exceção do tratamento com chlorimuron-ethyl, aplicado isoladamente, os demais tratamentos foram eficientes na dessecação de Brachiaria plantaginea.

Todos os tratamentos químicos foram eficientes na dessecação de Bidens pilosa.

Os herbicidas chlorimuron-ethyl, na dose de 10,0 g/ ha e diclosulam, na dose de 35,2 g/ha, proporcionaram efeito residual suficiente para controlar $B$. pilosa durante o ciclo da cultura.

\section{LITERATURA CITADA}

ASHTON, F. M.; CRAFTS, A. S. Mode of action of herbicides. New York: John Wiley, 1981. 525 p.

BIZZI, A. F.; BIANCHI, M. A. Eficácia de mistura de chlorimuron-ethyl com glyphosate na dessecação e residual sobre plantas daninhas na soja. In: CONGRESSO BRASILEIRO DA CIÊNCIA DAS PLANTAS DANINHAS, 22. Foz do Iguaçú, 2000. Resumos... Londrina: SBCPD, 2000. p. 148.

CARVALHO, F. T. de; MENDONÇA, M. R.; PERUCHI, M.; PALAZZO, R. R. B. Eficácia de herbicidas no manejo de Euphorbia heterophylla para o plantio direto de soja. Revista Brasileira de Herbicidas, v. 1, n. 1, p. 159$165,2000$.

LICH, J. M.; RENNER, K. A.; PENNER, D. Interaction of glyphosate with postemergence soybean (Glycine max) herbicides. Weed Science, v. 45, p. 12-21, 1997.

REUNIÃO DE PESQUISA DE SOJA DA REGIÃO SUL. Recomendações técnicas para a cultura da soja no Rio Grande do Sul e Santa Catarina 1994/95. Cruz Alta: Fundacep Fecotrigo, 1994. 66 p.

RODRIGUES, B. N. Guia de herbicidas. 3 ed. Londrina: Ed. dos Autores, 1995. 675 p.

ROMAN, E. S.; DIDONET, A. D. Controle de plantas daninhas no sistema de plantio direto de trigo e soja. Passo Fundo: EMBRAPA-CNPT, 1990. 32 p. (EMBRAPA-CNPT. Circular Técnica, 2).

THILL, D. C. Sulfonylureas and triazolopyrimidines. In: HERBICIDE ACTION COURSE, 1994. Summary of lectures. West Lafayette, Purdue University. p.317343.

VALENTE, T. O.; ORNELAS, A. Efeito residual de chlorimuron-ethyl aplicado em mistura com glyphosate na dessecação em condições de cerrado. In: CONGRESSO BRASILEIRO DA CIÊNCIA DAS PLANTAS DANINHAS, 22. Fóz do Iguaçú, 2000, Resumos... Londrina: SBCPD, 2000. p. 117.

ZIMDAHL, R. L. Fundamentals of weed science. San Diego: Academic Press, 1993. 450p. 
